\title{
Classes logarithmiques ambiges des corps quadratiques
}

\author{
par
}

Florence Soriano (Talence)

0. Introduction. La notion de corps logarithmiquement principal (relativement à un nombre premier $p$ ) a été introduite par J.-F. Jaulent à l'occasion de l'étude de certaines pro- $p$-extensions canoniques des corps de nombres en liaison avec la $K$-théorie : l'arithmétique des classes logarithmiques, qui se révèle semblable (quoique plus complexe) à celle des classes de diviseurs au sens habituel donne, en effet, des informations directes sur celle des noyaux modéré et sauvage des corps considérés : plus précisément, si $K$ désigne un corps de nombres qui contient les racines $2 l$-ièmes de l'unité, le $l$-groupe des classes logarithmiques s'interprête par la théorie d'Iwasawa comme le quotient des genres

$$
\widetilde{C l}_{K}={ }^{\Gamma} \mathcal{C}
$$

du groupe de Galois $\mathcal{C}=\operatorname{Gal}\left(\overline{K^{c}} / K^{\mathrm{c}}\right)$ attaché à la pro-l-extension abélienne complètement décomposée partout maximale $\bar{K}^{\mathrm{c}}$ du corps cyclotomique $K^{\mathrm{c}}=K\left[\zeta_{l^{\infty}}\right]$ relativement au groupe procyclique $\Gamma=\operatorname{Gal}\left(K^{\mathrm{c}} / K\right)\left(\right.$ cf. $\left.\left[\mathrm{J}_{1}\right]\right)$, tandis que la cohomologie galoisienne permet de montrer que la $l$-partie ${ }_{l} \infty H_{2}(K)$ du noyau des symboles de Hilbert dans $K_{2}(K)$ est donnée, elle, par l'identité (cf. [Sc], th. 7.3)

$$
{ }_{l} H_{2}(K)=\Gamma\left(\mathbb{T}_{l} \otimes_{\mathbb{Z}_{l}} \mathcal{C}\right),
$$

où $\mathbb{T}_{l}=\lim _{\longleftarrow} \mu_{l^{n}}$ désigne le module de Tate.

Il résulte de cela (cf., par exemple, $\left[\mathrm{J}_{2}\right]$, mais on peut aussi le montrer directement), que pour $l=2$ et en présence des racines 4-ièmes de l'unité, il existe un isomorphisme canonique

$$
\{ \pm 1\} \otimes_{\mathbb{Z}} \widetilde{C l}_{K}={ }^{2} H_{2}(K)
$$

entre les quotients d'exposant 2 respectifs du 2-groupe des classes logarithmiques et du noyau hilbertien de sorte en particulier que $\widetilde{C l}_{K}$ et ${ }_{2} \infty H_{2}(K)$ sont alors simultanément triviaux. 
Les résultats de J. Browkin et A. Schinzel (cf. [BS]) permettant de dresser la liste des extensions quadratiques $\mathbb{Q}(\sqrt{ \pm d})$ de $\mathbb{Q}$ pour lesquelles le 2-groupe ${ }_{2}{ }^{\infty} H_{2}(K)$ est trivial, il était tentant de regarder si la condition de trivialité ${ }_{2}^{\infty} H_{2}(K)=1$ était encore corrélée ou non avec la condition logarithmique analogue alors même que l'hypothèse $\mu_{4} \subset K$ n'était plus satisfaite. Nous nous proposons donc dans cet article de déterminer la liste des corps quadratiques $K=\mathbb{Q}(\sqrt{ \pm d})$ du corps des rationnels dont le 2-groupe des classes logarithmiques (au sens de $\left.\left[J_{3}\right]\right) \widetilde{C l}_{\mathbb{Q}(\sqrt{ \pm d})}$ est trivial et de comparer la classification obtenue à celle, tirée de [BS], des mêmes corps $K$, pour lesquelles c'est ${ }_{2 \infty} H_{2}(K)$ qui est trivial.

1. Notations et position du problème. Nous utilisons dans ce travail les notations de $\left[\mathrm{J}_{3}\right]$. En particulier nous posons :

1.1. DÉfinition. Le corps de nombres $K$ est dit 2-logarithmiquement principal si et seulement si son 2-groupe des classes logarithmiques $\widetilde{C l}_{K}$ est trivial.

Exemples. 1. Comme nous le verrons au paragraphe 5, l'exemple le plus simple est le corps des nombres rationnels $K=\mathbb{Q}$.

2. Si $i$ désigne une racine primitive quatrième de l'unité dans $\mathbb{C}$, le corps de nombres $K=\mathbb{Q}(i)$ étant 2-régulier (cf. par exemple, [GJ], Th. 2.1.iv.a.), son 2-noyau hilbertien $H_{2}(K)$ est en particulier trivial. Et ainsi, l'isomorphisme $\{ \pm 1\} \otimes_{\mathbb{Z}} \widetilde{C l}_{K}={ }_{2} H_{2}(K)$ montre que $K=\mathbb{Q}(i)$ est effectivement un corps de nombres 2-logarithmiquement principal .

Remarque. Le lien étroit entre la notion de groupe des classes logarithmiques avec celle du 2-Sylow du noyau hilbertien, nous suggère de citer certains auteurs dont les travaux concernent la trivialité du 2-noyau hilbertien des extensions quadratiques de $\mathbb{Q}$.

1. En 1972, les calculs de J. Tate (cf. [BT], appendice) montrent que le noyau hilbertien est trivial pour les corps quadratiques imaginaires dont le discriminant $\Delta$ de valeur absolue strictement inférieure à 35 n'est pas congru à 1 modulo 8 .

2. En 1982, J. Browkin et A. Schinzel publient dans [BS] les calculs du 2-rang du noyau hilbertien des extensions quadratiques de $\mathbb{Q}$.

3. En 1993, H. Qin (cf. [Qi]) prouve la trivialité du noyau hilbertien de $\mathbb{Q}(\sqrt{-6})$ par une méthode basée sur les travaux de Tate et transposable dans l'étude d'autres exemples de corps quadratiques imaginaires.

4. Un article récent de M. Skałba (cf. [Sk]) étudie les cas particuliers des extensions quadratiques imaginaires $\mathbb{Q}(\sqrt{-5})$ et $\mathbb{Q}(\sqrt{-19})$, comme application d'une généralisation du théorème de Thue. 
5. Enfin H. Thomas par des méthodes algorithmiques dresse dans [Th] la liste complète des extensions quadratiques de $\mathbb{Q}$ et de $\mathbb{Q}(i)$ dont le 2-noyau hilbertien est trivial.

Notre principal outil dans l'étude de la condition $\widetilde{C l}_{K}=0$ est l'inégalité des classes logarithmiques ambiges (cf. $\left[\mathrm{J}_{3}\right]$, Th. 4.5 ) qui établit en particulier qu'une extension quadratique $F$ du corps $\mathbb{Q}$ des nombres rationnels, de groupe de Galois $G$, est 2-logarithmiquement principale si et seulement si les deux conditions suivantes sont satisfaites :

(i) on a

$$
\prod_{\mathfrak{p} \mid \infty} d_{\mathfrak{p}}(F / \mathbb{Q}) \cdot \prod_{\mathfrak{p} \nmid \infty} \widetilde{e}_{\mathfrak{p}}(F / \mathbb{Q})=\left[F: F \cap \mathbb{Q}^{\mathrm{c}}\right] \cdot\left(\widetilde{\mathcal{E}}_{\mathbb{Q}}: \widetilde{\mathcal{E}}_{\mathbb{Q}} \cap \mathcal{N}_{F / \mathbb{Q}}\right)
$$

où $d_{\mathfrak{p}}(F / \mathbb{Q})$ désigne le degré local en $\mathfrak{p}$ de l'extension $F / \mathbb{Q}, \widetilde{e}_{\mathfrak{p}}(F / \mathbb{Q})$ le degré de ramification logarithmique, $\mathbb{Q}^{\mathrm{c}}$ la $\mathbb{Z}_{l}$-extension cyclotomique, $\widetilde{\mathcal{E}}_{\mathbb{Q}}$ le groupe des unités logarithmiques de $\mathbb{Q}$ et $\mathcal{N}_{L / K}$ le $\mathbb{Z}_{l}$-tensorisé des normes dans l'extension $F / \mathbb{Q}$;

(ii) le morphisme $\varphi$ du premier groupe de cohomologie $H^{1}\left(G, \widetilde{P l}_{F}\right)$ des diviseurs principaux dans celui $H^{1}\left(G, \widetilde{D l}_{F}\right)$ des diviseurs logarithmiques de degré nul de $F$, déduit de la suite exacte

$$
1 \rightarrow \widetilde{P l}_{F} \rightarrow \widetilde{D l}_{F} \rightarrow \widetilde{C l}_{F} \rightarrow 1,
$$

est surjectif.

Lors de l'étude de la surjectivité du morphisme $\varphi$, les lemmes 4.1 et 4.2 de $\left[J_{3}\right]$ nous conduisent à introduire la définition suivante :

1.2. Définition. Une 2-extension $L / K$ de corps de nombres est dite ( $C M)$-primitivement ramifiée (au sens logarithmique) si l'une au moins des conditions suivantes est vérifiée :

- l'extension $L / K$ est primitivement ramifiée, c'est-à-dire les diviseurs logarithmiques de $L$ sont sommes de diviseurs ambiges et de diviseurs de degré nul,

- le quotient $\widetilde{\mathcal{E}}_{K} \cap \mathcal{N}_{L / K} / N_{L / K}\left(\widetilde{\mathcal{E}}_{L}\right)$ est engendré par des éléments totalement positifs (on parle alors d'extension $(C M)$ )-primitivement ramifiée au sens non trivial).

Remarque. Comme $\mathbb{Q}$ ne possède qu'une place paire, le groupe $\widetilde{\mathcal{E}}_{\mathbb{Q}}$ des unités logarithmiques de $\mathbb{Q}$ coïncide avec le tensorisé du groupe des 2 -unités logarithmiques de $\mathbb{Q}$, et est en particulier engendré par -1 et 2 . Les 2-extensions $(C M)$-primitivement ramifiées de $\mathbb{Q}$ comprennent en particulier les extensions quadratiques imaginaires de $\mathbb{Q}$, et les extensions quadratiques réelles dont les unités -1 et -2 ne sont pas normes. 
Sous l'hypothèse de $(C M)$-primitivité, les deux paragraphes suivants établissent qu'en fait le morphisme $\varphi$ est surjectif si et seulement si ni le radical $\pm d$, ni sa moitié $\pm d / 2$ ne sont congrus à 9 modulo 16 . Autrement dit, l'ordre du 2-groupe des classes logarithmiques ambiges de l'extension quadratique $F=\mathbb{Q}(\sqrt{ \pm d})$ de $\mathbb{Q}$ est donné dans chacun des cas suivants par la formule :

$$
\begin{gathered}
1^{\text {er } c a s: \text { pour }} \pm d \equiv 1(\bmod 16) \text { ou } \pm d \equiv 2(\bmod 32), \text { on a } \\
\left|\widetilde{C l} l_{F}^{G}\right|=\frac{\prod_{\mathfrak{p} \mid \infty} d_{\mathfrak{p}}(F / \mathbb{Q}) \cdot \prod_{\mathfrak{p} \nmid \infty} \widetilde{e}_{\mathfrak{p}}(F / \mathbb{Q})}{\left[F: F \cap \mathbb{Q}^{\mathrm{c}}\right] \cdot\left(\widetilde{\mathcal{E}}_{\mathbb{Q}}: \widetilde{\mathcal{E}}_{\mathbb{Q}} \cap \mathcal{N}_{F / \mathbb{Q}}\right)}\left|H^{1}\left(G, \widetilde{D l} l_{F}\right)\right|, \\
2^{\text {nd }} \text { cas : pour } \pm d \neq \equiv 1(\bmod 16) \text { et } \pm d \not \equiv 2(\bmod 32) \text {, on a } \\
\left|\widetilde{C l} l_{F}^{G}\right|=\frac{\prod_{\mathfrak{p} \mid \infty} d_{\mathfrak{p}}(F / \mathbb{Q}) \cdot \prod_{\mathfrak{p} \nmid \infty} \widetilde{e}_{\mathfrak{p}}(F / \mathbb{Q})}{\left[F: F \cap \mathbb{Q}^{c}\right] \cdot\left(\widetilde{\mathcal{E}}_{\mathbb{Q}}: \widetilde{\mathcal{E}}_{\mathbb{Q}} \cap \mathcal{N}_{F / \mathbb{Q}}\right)} .
\end{gathered}
$$

La liste des extensions quadratiques 2-logarithmiquement principales $F$ de $\mathbb{Q}$ est alors dans ce cas facilement déterminée, et fait l'objet du paragraphe 6 .

\section{Classes logarithmiques ambiges des extensions quadratiques de $\mathbb{Q}$}

2.1. Présentation du problème. Comme nous l'avons dit, nous souhaitons calculer l'ordre du groupe des classes logarithmiques ambiges d'une extension quadratique $F$ quelconque de $K=\mathbb{Q}$. La formule des classes logarithmiques ambiges permettant de conclure dans le cas primitif (i.e. lorsque $H^{1}\left(G, \widetilde{D l}_{F}\right)=1$ (cf. $\left.\left.\S 3\right)\right)$, le problème est dans le cas contraire ramené à l'étude de la surjectivité du morphisme $\varphi: H^{1}\left(G, \widetilde{P l}_{F}\right) \rightarrow H^{1}\left(G, \widetilde{D l}_{F}\right)$, déduit de la suite exacte

$$
1 \rightarrow \widetilde{P l}_{F} \rightarrow \widetilde{D l}_{F} \rightarrow \widetilde{C l}_{F} \rightarrow 1
$$

D'après $\left[\mathrm{J}_{3}\right], \S 4$, le nombre de classes logarithmiques ambiges est donné, en effet, par la formule

$$
\left|\widetilde{C l}_{F}^{G}\right|=\left|\widetilde{C l}_{\mathbb{Q}}\right| \frac{\prod_{\mathfrak{p} \mid \infty} d_{\mathfrak{p}}(F / \mathbb{Q}) \cdot \prod_{\mathfrak{p} \nmid \infty} \widetilde{e}_{\mathfrak{p}}(F / \mathbb{Q})}{\left[F: F \cap \mathbb{Q}^{c}\right] \cdot\left(\widetilde{\mathcal{E}}_{\mathbb{Q}}: \widetilde{\mathcal{E}}_{\mathbb{Q}} \cap \mathcal{N}_{F / \mathbb{Q}}\right)}|\operatorname{Coker} \varphi| .
$$

La difficulté majeure de manipulation de cette formule réside donc en l'étude de la surjectivité du morphisme de groupes cohomologiques $\varphi$. Cette étude est bien entendu plus facile lorsque le premier groupe cohomologique $H^{1}\left(G, \widetilde{P l}_{F}\right)$ est trivial, puisque le nombre de classes logarithmiques ambiges de $F$ est donné alors par la relation

$$
\left|\widetilde{C l}_{F}^{G}\right|=\left|\widetilde{C l}_{\mathbb{Q}}\right| \frac{\prod_{\mathfrak{p} \mid \infty} d_{\mathfrak{p}}(F / \mathbb{Q}) \cdot \prod_{\mathfrak{p} \nmid \infty} \widetilde{e}_{\mathfrak{p}}(F / \mathbb{Q})}{\left[F: F \cap \mathbb{Q}^{c}\right] \cdot\left(\widetilde{\mathcal{E}}_{\mathbb{Q}}: \widetilde{\mathcal{E}}_{\mathbb{Q}} \cap \mathcal{N}_{F / \mathbb{Q}}\right)}\left|H^{1}\left(G, \widetilde{D l} l_{F}\right)\right| .
$$


Pour ces raisons, au moins dans le cas imprimitif, l'étude de la surjectivité de $\varphi$ est ramenée à celle de la trivialité de l'image d'une classe génératrice du groupe $H^{1}\left(G, \widetilde{P l}_{F}\right)$ (d'ordre 1 ou 2). D'où la nécessité dans un premier temps, de revenir sur la définition de $\varphi$ :

2.2. Etude du morphisme $\varphi$. Le diagramme commutatif suivant (où tous les groupes sont d'ordre 1 ou 2) :

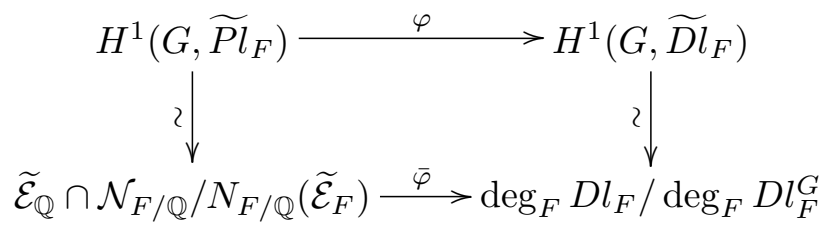

déduit des isomorphismes donnés par les lemmes 4.1 et 4.2 dans $\left[\mathrm{J}_{3}\right]$, montre que les morphismes $\varphi$ et $\bar{\varphi}$ sont équivalents. Plus précisément :

2.2.1. Proposition. Soient $\mathbb{Q}$ le corps des nombres rationnels et $F$ une extension quadratique de $\mathbb{Q}$, dont le groupe de Galois $G$ est engendré par un $\mathbb{Q}$-automorphisme $\sigma$. Le morphisme $\varphi$ du premier groupe de cohomologie $H^{1}\left(G, \widetilde{P l}_{F}\right)$ des diviseurs principaux de $F$ dans le premier groupe de cohomologie $H^{1}\left(G, \widetilde{D l}_{F}\right)$ des diviseurs de degré nul de $F$ induit un morphisme $\bar{\varphi}$ qui à toute classe dans $\widetilde{\mathcal{E}}_{\mathbb{Q}} \cap \mathcal{N}_{F / \mathbb{Q}} / N_{F / \mathbb{Q}}\left(\widetilde{\mathcal{E}}_{F}\right)$ d'une unité logarithmique $N_{F / \mathbb{Q}}(\alpha)$, norme de l'extension $F / \mathbb{Q}$, associe la classe dans le groupe quotient $\operatorname{deg}_{F} D l_{F} / \operatorname{deg}_{F} D l_{F}^{G}$, du degré d'un diviseur $\mathfrak{A}$ tel que $\mathfrak{A}^{\sigma}-\mathfrak{A}=$ $\widetilde{\operatorname{div}}_{F}(\alpha)$.

Preuve. Si $N_{F / \mathbb{Q}}(\alpha)$ désigne une unité logarithmique du corps $\mathbb{Q}$ des nombres rationnels, norme de l'extension $F / \mathbb{Q}$, il est toujours possible de déterminer un diviseur $\mathfrak{A}$ de $F$ tel que $\mathfrak{A}^{\sigma}-\mathfrak{A}=\widetilde{\operatorname{div}}_{F}(\alpha)$. En effet, la condition normique $N_{F / \mathbb{Q}}(\alpha) \in \widetilde{\mathcal{E}}_{\mathbb{Q}}$, qui s'écrit encore $\widetilde{\operatorname{div}_{\mathbb{Q}}} N_{F / \mathbb{Q}}(\alpha)=0$, nous donne les conditions

$$
\widetilde{v}_{\mathfrak{p}}(\alpha)+\widetilde{v}_{\mathfrak{p}}^{\sigma}(\alpha)=0, \quad \forall \mathfrak{p} \in P l_{F}^{\circ} \text { (ensemble des places finies de } F \text { ). }
$$

Faisons donc choix d'un système de représentants $P l_{F / \mathbb{Q}}^{\circ}$ de $P l_{F}^{\circ}$ pour l'action du groupe de Galois $G=\operatorname{Gal}(F / \mathbb{Q})$ (autrement dit, pour chaque premier $p$ de $\mathbb{Q}$ décomposé dans $F / \mathbb{Q}$, faisons choix de l'un des premiers $\mathfrak{p}$ de $F$ au-dessus de $p$ ), et considérons le diviseur logarithmique

$$
\mathfrak{A}=-\sum_{\mathfrak{p} \in P l_{F / \mathbb{Q}}^{\circ}} \widetilde{v}_{\mathfrak{p}}(\alpha) \mathfrak{p} .
$$

Nous obtenons immédiatement

$$
\mathfrak{A}^{\sigma}-\mathfrak{A}=\sum_{\mathfrak{p} \in P l_{F / \mathbb{Q}}^{\circ}} \widetilde{v}_{\mathfrak{p}}(\alpha)\left(\mathfrak{p}-\mathfrak{p}^{\sigma}\right),
$$




$$
\begin{aligned}
\mathfrak{A}^{\sigma}-\mathfrak{A} & =\sum_{\mathfrak{p} \in P l_{F / \mathbb{Q}}^{\circ}}\left(\widetilde{v}_{\mathfrak{p}}(\alpha) \mathfrak{p}-\widetilde{v}_{\mathfrak{p}}(\alpha) \mathfrak{p}^{\sigma}\right)=\sum_{\mathfrak{p} \in P l_{F / \mathbb{Q}}^{\circ}}\left(\widetilde{v}_{\mathfrak{p}}(\alpha) \mathfrak{p}+\widetilde{v}_{\mathfrak{p}}^{\sigma}(\alpha) \mathfrak{p}^{\sigma}\right) \\
& =\widetilde{\operatorname{div}}_{F}(\alpha),
\end{aligned}
$$

comme attendu.

Deux étapes s'imposent alors :

(i) montrer que l'image $\operatorname{deg} \mathfrak{A}\left(\bmod D l_{F}^{G}\right)$ de $\mathfrak{A}$ par $\bar{\varphi}$ ne dépend en fait que de l'unité logarithmique de départ $N_{F / \mathbb{Q}}(\alpha)$,

(ii) montrer que cette image est nulle dès que $\alpha$ est une unité logarithmique de $F$.

Ainsi, dans un premier temps, nous considérons $\mathfrak{A}$ et $\mathfrak{B}$, deux diviseurs tels que $\mathfrak{A}^{\sigma}-\mathfrak{A}=\mathfrak{B}^{\sigma}-\mathfrak{B}=\widetilde{\operatorname{div}}_{F}(\alpha)$. L'écriture du diviseur $\mathfrak{A}^{\sigma}-\mathfrak{B}^{\sigma}$ sous la forme

$$
\begin{aligned}
\mathfrak{A}^{\sigma}-\mathfrak{B}^{\sigma} & =(\mathfrak{A}-\mathfrak{B})+\left(\mathfrak{A}^{\sigma}-\mathfrak{A}\right)+\left(\mathfrak{B}-\mathfrak{B}^{\sigma}\right) \\
& =(\mathfrak{A}-\mathfrak{B})+\widetilde{\operatorname{div}}_{F}(\alpha)-\widetilde{\operatorname{div}}_{F}(\alpha)=\mathfrak{A}-\mathfrak{B}
\end{aligned}
$$

montre que $\mathfrak{A}-\mathfrak{B}$ est un diviseur ambige et donc que les degrés de $\mathfrak{A}$ et $\mathfrak{B}$ ont même image dans le premier groupe de cohomologie $H^{1}\left(G, \widetilde{D l}_{F}\right) \simeq$ $\operatorname{deg}_{F} D l_{F} / \operatorname{deg}_{F} D l_{F}^{G}$. Enfin, soit $\mathfrak{A}$ un diviseur logarithmique provenant, par la construction précédente, de la norme d'une unité logarithmique de $F$; alors, conservant les notations, nous obtenons banalement $\mathfrak{A}=0$ donc trivialement $\operatorname{deg}_{F} \mathfrak{A}=0$.

Tous les outils de manipulation enfin présentés, commençons par examiner le cas le plus simple correspondant aux extensions primitivement ramifiées.

3. Application au cas primitif. Ce chapitre vise à caractériser les extensions quadratiques $F=\mathbb{Q}(\sqrt{ \pm d})$ primitivement ramifiées (i.e. telles que $\left.H^{1}\left(G, \widetilde{D l}_{F}\right)=1\right)$. Plus précisément, il établit le résultat suivant :

3.1. LemME. L'entier naturel d étant supposé sans facteur carré, l'extension quadratique $\mathbb{Q}(\sqrt{ \pm d}) / \mathbb{Q}$ est primitivement ramifiée si et seulement si $\pm d$ n'est congru ni à 1 modulo 8 , ni à 2 modulo 16 , ou encore si d est divisible par un premier impair $p$ congru à \pm 3 modulo 8 , ou enfin si $\pm d=2$.

Preuve. Si $\pm d=2$, les corps de nombres $F$ et $F \cap \mathbb{Q}^{c}$ coïncident, si bien que l'extension est trivialement primitivement ramifiée.

Sinon, il s'agit de vérifier l'existence d'un diviseur premier $p$ ramifié au sens logarithmique dans l'extension $F / F \cap \mathbb{Q}^{c}$, et dont le degré est générateur du $\mathbb{Z}_{2}$-module $\operatorname{deg}_{\mathbb{Q}} D l_{\mathbb{Q}}=4 \mathbb{Z}_{2}$. Dans le cas de ramification logarithmique en 2 , c'est-à-dire $\pm d \not \equiv 1(\bmod 8)$ et $\pm d \not \equiv 2(\bmod 16)$, l'extension $F / \mathbb{Q}$ est 
primitivement ramifiée (puisque le degré $\operatorname{deg}_{F} \mathcal{L}$ d'une place sauvage $\mathcal{L}$ de $F$ est égal à 4$)$.

Dans le cas $\pm d \equiv 1(\bmod 8)$ ou $\pm d \equiv 2(\bmod 16)$, l'extension $F / \mathbb{Q}$ ne peut être primitivement ramifiée qu'en un diviseur premier $p$ de $\mathbb{Q}$, divisant l'entier $d$. De plus, un diviseur premier impair $p$ de $\mathbb{Q}$ est primitif si et

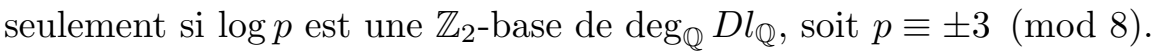

Remarque. Dans cette situation, le conoyau de $\varphi$ est clairement trivial, si bien que la formule des classes logarithmiques ambiges est aisément applicable.

4. Application au cas ( $C M)$-primitif. Nous notons $F$ une extension quadratique de $\mathbb{Q}$, que nous supposons dans ce paragraphe $(C M)$ primitivement ramifiée (au sens non trivial), si bien que $F$ est une extension non primitivement ramifiée et de la forme $\mathbb{Q}(\sqrt{ \pm d})$, où $d$ est un entier sans facteur carré et strictement supérieur à 2 .

Ce chapitre se restreignant au cas imprimitif (sinon cf. §3), nous avons les congruences

$$
\pm d \equiv 1(\bmod 8) \quad \text { ou } \quad \pm d \equiv 2(\bmod 16) .
$$

L'une des conséquences immédiates de cette hypothèse de $(C M)$-primitivité au sens non trivial est, comme nous allons le voir, l'existence d'un élément $\alpha$ de norme 2 dans l'extension $F / \mathbb{Q}$.

4.1. Lemme. Si $F / \mathbb{Q}$ désigne une extension quadratique de $\mathbb{Q}$, que nous supposons (CM)-primitivement ramifiée au sens non trivial, alors l'unité logarithmique 2 est norme dans l'extension $F / \mathbb{Q}$.

Preuve. Soit $d$ l'entier positif, supposé sans facteur carré, tel que $F=\mathbb{Q}(\sqrt{ \pm d})$. Dire que 2 est norme dans l'extension $F / \mathbb{Q}$, c'est dire que les symboles quadratiques de Hilbert $[2, \pm d]_{p}$ attachés aux places $p$ de $\mathbb{Q}$ valent tous +1 . Lorsque $p$ est infini ou étranger à $d$, le symbole $[2, \pm d]_{p}$ est naturellement trivial. Enfin, lorsque $p$ désigne un diviseur premier impair de $d, p$ est congru à \pm 1 modulo 8 (puisque l'extension $F / \mathbb{Q}$ n'est pas primitivement ramifiée), si bien que

$$
[2, \pm d]_{p}=\left(\frac{2}{p}\right)=1 .
$$

La formule du produit $\prod_{p \in P}[2, \pm d]_{p}=1$, où $P$ désigne l'ensemble des places de $\mathbb{Q}$, établit la trivialité de l'expression $[2, \pm d]_{2}$. Par conséquent, 2 est norme locale partout dans l'extension $\mathbb{Q}(\sqrt{ \pm d}) / \mathbb{Q}$ et donc, par le principe de Hasse, norme globale.

Enfin, en notant que le groupe $\widetilde{\mathcal{E}}_{\mathbb{Q}}$ des unités logarithmiques de $\mathbb{Q}$ est engendré par les entiers -1 et 2 , et en nous plaçant sous la condition de 
$(C M)$-primitivité au sens logarithmique, nous pouvons convenir que 2 est un représentant d'une classe génératrice du groupe (éventuellement trivial) $H^{1}\left(G, \widetilde{P l}_{F}\right)$. Toutes les hypothèses restrictives ayant été faites, nous allons chercher à déterminer l'image par $\varphi$ de la classe de 2 dans $H^{1}\left(G, \widetilde{P l}_{F}\right)$.

Dire que 2 est norme dans l'extension quadratique $\mathbb{Q}(\sqrt{ \pm d}) / \mathbb{Q}$ revient évidemment à affirmer que $\pm d$ est norme dans l'extension quadratique $\mathbb{Q}(\sqrt{2}) / \mathbb{Q}$, c'est-à-dire, puisque l'anneau $\mathbb{Z}[\sqrt{2}]$ est principal, qu'il existe deux entiers relatifs $u$ et $v$ tels que l'on ait $u^{2}-2 v^{2}= \pm d$. En effet, si $\pm d$ est la norme de l'élément $\alpha$ dans l'extension $\mathbb{Q}(\sqrt{2}) / \mathbb{Q}$, alors $\alpha$ s'écrit sous la forme $q \times \prod_{p \in I} \pi_{p}^{n_{p}} \bar{\pi}_{p}^{n_{p}^{\prime}}$ où $q$ est un rationnel ne faisant intervenir que les premiers $p$ non décomposés dans l'extension $\mathbb{Q}(\sqrt{2}) / \mathbb{Q}$ et $I$ désigne l'ensemble des premiers $p$ se décomposant en deux irréductibles notés $\pi_{p}$ et $\bar{\pi}_{p}$. L'identité $N_{L / K}\left(\pi_{p} / \bar{\pi}_{p}\right)=1$ nous permet de supposer que les entiers $n_{p}$ et $n_{p}^{\prime}$ sont de même signe. Cela étant, le radical $\pm d$ étant supposé sans facteur carré, le passage à la norme

$$
\pm d=N_{L / K}(\alpha)=q^{2} \times \prod_{p \in I} p^{n_{p}+n_{p}^{\prime}}
$$

nous donne alors $q= \pm 1$ et $n_{p}+n_{p}^{\prime}=0$ ou 1 , si bien que $\alpha$ est alors un entier de $\mathbb{Z}[\sqrt{2}]$.

Introduisons maintenant les nouvelles notations suivantes :

- $\alpha=(u+\sqrt{ \pm d}) / v$ et $\beta=u+\sqrt{ \pm d}$,

- $\bar{\alpha}$ et $\bar{\beta}$ les conjugués respectifs de $\alpha$ et $\beta$.

Nous avons par construction (en abrégeant $N_{F / \mathbb{Q}}$ par $N$ )

$$
N(\beta)=\beta \bar{\beta}=(u+\sqrt{ \pm d})(u-\sqrt{ \pm d})=u^{2} \mp d=2 v^{2},
$$

et par suite,

$$
N(\alpha)=N\left(\frac{\beta}{v}\right)=\frac{N(\beta)}{v^{2}}=2,
$$

comme attendu. L'étude de la surjectivité de $\bar{\varphi}$ nous suggère l'étude de la parité des $\mathfrak{p}$-valuations logarithmiques de $\beta$.

4.2. Lemme. En conservant les hypothèses et notations précédentes, les $\mathfrak{p}$-valuations logarithmiques de $\beta=u+\sqrt{ \pm d}$ sont paires en toute place finie modérée (i.e. impaire) $\mathfrak{p}$ de $F$.

Preuve. Nous avons le système suivant :

$$
\begin{aligned}
\beta \bar{\beta} & =2 v^{2}, \\
\beta+\bar{\beta} & =2 u, \\
\beta-\bar{\beta} & =2 \sqrt{ \pm d}, \\
2 v^{2} & =u^{2} \mp d .
\end{aligned}
$$


Plaçons nous dans le cas où $\mathfrak{p}$ désigne une place de $F$, au-dessus d'un nombre premier impair $p$, et raisonnons par l'absurde, en supposant que la $\mathfrak{p}$-valuation de $\beta$ est impaire. Sous ces hypothèses, $\mathfrak{p}$ divise $\beta$. Or, les $\mathfrak{p}$-valuations de $\beta$ et $\bar{\beta}$ ayant même parité (d'après (1)), p divise aussi $\bar{\beta}$, donc $u$ et $\sqrt{ \pm d}$ (avec (2) et (3)), et enfin $v$ (avec (4)). Ainsi $p$ divise $u$ et $v$, ce qui implique d'après (4) que le carré $p^{2}$ divise l'entier $d$, supposé sans facteur carré.

Lorsque $\mathfrak{p}$ désigne une place sauvage $\mathcal{L}$, deux cas interviennent :

4.3. Le cas décomposé $\pm d \equiv 1(\bmod 8)$. Dans le cas $\pm d \equiv 1(\bmod 8)$ (où l'extension locale $F_{\mathcal{L}} / \mathbb{Q}_{2}$ est triviale), nous imposons, pour des raisons techniques, quelques conditions supplémentaires aux entiers $u$ et $v$.

4.3.1. Lemme. Soient $F$ une extension quadratique de $\mathbb{Q}(C M)$-primitivement ramifiée (au sens non trivial) et d l'entier naturel, supposé sans facteur carré, tel que $F=\mathbb{Q}(\sqrt{ \pm d})$. En conservant les notations précédemment précisées, il est toujours possible, lorsque l'entier $\pm d$ est congru à 1 modulo 8, de choisir les entiers $u$ et $v$ satisfaisant les deux propriétés suivantes :

$$
\left\{\begin{array}{l}
u^{2}-2 v^{2}= \pm d \\
2 u \mp d+3 \in 32 \mathbb{Z} .
\end{array}\right.
$$

Preuve. Ecrivons $\pm d=u^{2}-2 v^{2}$ pour deux entiers $u$ et $v$. De la congruence $\pm d \equiv 1(\bmod 8)$, nous concluons que $u$ est impair, ce qui nous donne $u^{2} \equiv 1(\bmod 8)$; il suit que $v$ est pair, autrement dit $v \equiv 0(\bmod 4)$ ou $v \equiv 2(\bmod 4)$.

- $1^{\mathrm{er}}$ cas : pour $v \equiv 0(\bmod 4)$, nous pouvons, quitte à changer $u$ en $-u$, supposer $u \equiv-1(\bmod 4)$.

- $2^{\text {ième }}$ cas : pour $v \equiv 2(\bmod 4)$, choisissons $u \equiv 1(\bmod 4)$, et considérons le couple $\left(u^{\prime}, v^{\prime}\right)$ défini par

$$
u^{\prime}+v^{\prime} \sqrt{2}=(u+v \sqrt{2})(1+\sqrt{2})^{2} .
$$

Nous avons encore $\pm d=u^{\prime 2}-2 v^{\prime 2}$ avec, maintenant

$$
\left\{\begin{array}{l}
u^{\prime}=3 u+4 v \equiv-1(\bmod 4), \\
v^{\prime}=2 u+3 v \equiv 0(\bmod 4)
\end{array}\right.
$$

et le remplacement du couple $(u, v)$ par le couple $\left(u^{\prime}, v^{\prime}\right)$ nous ramène au cas précédent.

Cela acquis, écrivons donc $\pm d=u^{2}-2 v^{2}$ avec $u \equiv-1(\bmod 4)$, disons $u=-1+4 a$, et $v \equiv 0(\bmod 4)$, disons $v=4 b$. Nous obtenons, comme annoncé,

$$
\begin{gathered}
2 u \mp d+3=2 u-u^{2}+2 v^{2}+3=-2+8 a-1+8 a-16 a^{2}+32 b^{2}+3, \\
2 u \mp d+3=-16 a(a-1)+32 b^{2} \equiv 0(\bmod 32) .
\end{gathered}
$$


L'intérêt de la construction d'un tel élément $\alpha$ provient du lemme suivant :

4.3.2. Lemme. Sous l'hypothèse de (CM)-primitivité au sens non trivial, il existe un diviseur logarithmique $\mathfrak{B}$ de degré nul tel que

$$
\widetilde{\operatorname{div}}_{F}(\beta)=2 \mathfrak{B}+\varrho\left(\mathcal{L}-\mathcal{L}^{\sigma}\right)
$$

où @ est l'entier $0($ resp. 1$)$ si $\pm d \equiv 1(\bmod 16)($ resp. $\pm d \equiv 9(\bmod 16))$.

Preuve. Compte tenu du lemme 4.2, il s'agit ici d'examiner la $\mathcal{L}$-valuation logarithmique de $\beta$ :

- $\mathrm{Si} \pm d \equiv 1(\bmod 16)$, les complétés en 2 des corps de nombres $F$ et $\mathbb{Q}$ coïncident. Nous sommes alors amenés à faire choix d'une racine de $\pm d$, que nous noterons $\delta$ et prendrons congrue à -1 modulo 8 . Par suite, il vient successivement :

$$
\begin{aligned}
(\delta+1)^{2} & \equiv 1+2 \delta+\delta^{2} \equiv 0(\bmod 64) \\
\delta & \equiv-\frac{ \pm d+1}{2}(\bmod 32) \\
\delta+u & \equiv \frac{2 u \mp d-1}{2}(\bmod 32) .
\end{aligned}
$$

C'est ici qu'intervient la troisième propriété de $d$, puisqu'elle nous permet d'écrire $2 u \mp d-1 \equiv-4(\bmod 32)$, et donc d'établir la parité de la $\mathcal{L}$ valuation logarithmique de $\beta$.

- Si $\pm d \equiv 9(\bmod 16)$, nous faisons alors choix d'une racine de $\pm d$, que nous noterons encore $\delta$ et prendrons congrue à 3 modulo 8. Par suite, $\delta+u \equiv(6 u \pm d+9) / 6(\bmod 32)$.

Comme $6 u \pm d+9=3(2 u \mp d+3)+4 d$, nous avons $6 u \pm d+9 \equiv$ $4(\bmod 32)$, soit

$$
6 u \pm d+9 \in 2^{\mathbb{Z}} \times\left( \pm 1+8 \mathbb{Z}_{2}\right),
$$

et enfin, $\delta+u \in 2^{\mathbb{Z}} \times\left( \pm 3+8 \mathbb{Z}_{2}\right)$. Ceci prouve que la $\mathcal{L}$-valuation logarithmique de $\beta$ est effectivement impaire. Dans les deux cas, $\mathfrak{B}=\frac{1}{2}\left(\widetilde{\operatorname{div}}_{F}(\beta)-\right.$ $\left.\varrho\left(\mathcal{L}-\mathcal{L}^{\sigma}\right)\right)$ est effectivement un diviseur logarithmique de degré nul.

Nous concluons alors par le résultat suivant :

4.3.3. Proposition. Soient $F$ une extension quadratique de $\mathbb{Q}(C M)$ primitivement ramifiée et d l'entier naturel, supposé sans facteur carré, tel que $F=\mathbb{Q}(\sqrt{ \pm d})$ sous la contrainte $\pm d \equiv 1(\bmod 8)$. En désignant par $G$ le groupe de Galois de l'extension $F / \mathbb{Q}$, on trouve le nombre de classes logarithmiques ambiges de $F$ donné selon le cas par la formule correspondante: 
- ou bien $\pm d \equiv 1(\bmod 16)$, auquel cas

$$
\left|\widetilde{C l}_{F}^{G}\right|=\left|\widetilde{C l}_{\mathbb{Q}}\right| \frac{\prod_{\mathfrak{p} \mid \infty} d_{\mathfrak{p}}(F / \mathbb{Q}) \cdot \prod_{\mathfrak{p} \nmid \infty} \widetilde{e}_{\mathfrak{p}}(F / \mathbb{Q})}{\left[F: F \cap \mathbb{Q}^{\mathrm{c}}\right] \cdot\left(\widetilde{\mathcal{E}}_{\mathbb{Q}}: \widetilde{\mathcal{E}}_{\mathbb{Q}} \cap \mathcal{N}_{F / \mathbb{Q}}\right)}\left|H^{1}\left(G, \widetilde{D l} l_{F}\right)\right| ;
$$

- ou bien $\pm d \equiv 9(\bmod 16)$, auquel cas

$$
\left|\widetilde{C l}_{F}^{G}\right|=\left|\widetilde{C l}_{\mathbb{Q}}\right| \frac{\prod_{\mathfrak{p} \mid \infty} d_{\mathfrak{p}}(F / \mathbb{Q}) \cdot \prod_{\mathfrak{p} \nmid \infty} \widetilde{e}_{\mathfrak{p}}(F / \mathbb{Q})}{\left[F: F \cap \mathbb{Q}^{\mathrm{c}}\right] \cdot\left(\widetilde{\mathcal{E}}_{\mathbb{Q}}: \widetilde{\mathcal{E}}_{\mathbb{Q}} \cap \mathcal{N}_{F / \mathbb{Q}}\right)} .
$$

Preuve. Avec les notations du lemme 4.3.2, nous obtenons

$$
2\left(\mathfrak{B}+\mathfrak{B}^{\sigma}\right)=\widetilde{\operatorname{div}}_{F}(N \beta)=\widetilde{\operatorname{div}}_{F}\left(v^{2}\right)=2 \widetilde{\operatorname{div}}_{F}(v),
$$

si bien que

$$
\mathfrak{B}+\mathfrak{B}^{\sigma}=\widetilde{\operatorname{div}}_{F}(v)
$$

puis

$$
\begin{aligned}
\widetilde{\operatorname{div}}_{F}(\alpha) & =\widetilde{\operatorname{div}}_{F}(\beta / v)=\widetilde{\operatorname{div}}_{F}(\beta)-\widetilde{\operatorname{div}}_{F}(v) \\
& =2 \mathfrak{B}+\varrho\left(\mathcal{L}-\mathcal{L}^{\sigma}\right)-\left(\mathfrak{B}+\mathfrak{B}^{\sigma}\right)=\mathfrak{B}-\mathfrak{B}^{\sigma}+\varrho\left(\mathcal{L}-\mathcal{L}^{\sigma}\right)
\end{aligned}
$$

ce qui nous invite à poser $\mathfrak{A}=\mathfrak{B}^{\sigma}+\varrho \mathcal{L}^{\sigma}$. Il vient ainsi $\widetilde{\operatorname{div}_{F}}(\alpha)=\mathfrak{A}^{\sigma}-\mathfrak{A}$ avec $\operatorname{deg}_{F} \mathfrak{A}=\varrho \operatorname{deg}_{F} \mathcal{L}^{\sigma}=\varrho \operatorname{deg}_{F} \mathcal{L}$, soit selon le reste modulo 16 de $\pm d$,

$$
\operatorname{deg}_{F} \mathfrak{A}= \begin{cases}0 & \text { si } \pm d \equiv 1(\bmod 16), \\ \operatorname{deg}_{F} \mathcal{L} & \text { si } \pm d \equiv 9(\bmod 16) .\end{cases}
$$

Dans le premier cas, le morphisme $\varphi$ est nul, si bien qu'il ne peut être surjectif. Dans le second cas, le $\mathbb{Z}_{2}$-module des degrés des diviseurs logarithmiques de $F$ coïncidant avec le $\mathbb{Z}_{2}$-module $4\left[F \cap \mathbb{Q}^{\mathrm{c}}: \mathbb{Q}\right] \mathbb{Z}_{2}=4 \mathbb{Z}_{2}$, le $\mathbb{Z}_{2}$-module des degrés des diviseurs ambiges est en fait le $\mathbb{Z}_{2}$-module $8 \mathbb{Z}_{2}$ puisque l'extension $F / \mathbb{Q}$ est par hypothèse non primitivement ramifiée. Comme le degré de la place sauvage $\mathcal{L}$ est égale à 4 , le degré de $\mathcal{L}$ est générateur du $\mathbb{Z}_{2}$-module $\operatorname{deg}_{F} D l_{F}$ si bien que l'image par $\varphi$ de l'unité logarithmique 2 n'est pas nulle. Le morphisme $\varphi$ est donc ici surjectif.

4.4. Le cas non décomposé $d \equiv 2(\bmod 16)$. Le cas non décomposé se traite de façon analogue; nous en donnons cependant les différentes étapes.

4.4.1. Lemme. Sous l'hypothèse de (CM)-primitivité au sens non trivial, il existe un diviseur logarithmique $\mathfrak{B}$ de degré nul tel que

$$
\widetilde{\operatorname{div}}_{F}(\beta)=2 \mathfrak{B}+\varrho \mathcal{L}
$$

où $\varrho$ est l'entier égal à 0 (resp. 1) si $\pm d \equiv 2(\bmod 32)$ (resp. $\pm d \equiv$ $18(\bmod 32))$.

Preuve. Comme pour le lemme 4.3.2, nous examinons la $\mathcal{L}$-valuation logarithmique du nombre $\beta$, qui par définition, est paire si et seulement si 
la norme $2 v^{2}$ de $\beta=u+v \sqrt{ \pm d}$ appartient à $\{ \pm 1\} \times 2^{\mathbb{Z}} \times\left(1+16 \mathbb{Z}_{2}\right)$. La relation $u^{2}-2 v^{2}= \pm d$ nous impose les congruences

$$
u^{2} \equiv 4(\bmod 32), \quad v^{2} \equiv \begin{cases}1(\bmod 16) & \text { si } \pm d \equiv 2(\bmod 32) \\ 9(\bmod 16) & \text { si } \pm d \equiv 18(\bmod 32)\end{cases}
$$

ce qui achève la démonstration.

Nous concluons là aussi par le résultat analogue :

4.4.2. Proposition. Soient $F$ une extension quadratique de $\mathbb{Q}(C M)$ primitivement ramifiée et d l'entier naturel, supposé sans facteur carré, tel que $F=\mathbb{Q}(\sqrt{ \pm d})$ sous la contrainte $\pm d \equiv 2(\bmod 16)$. En désignant par $G$ le groupe de Galois de l'extension $F / \mathbb{Q}$, on trouve le nombre de classes logarithmiques ambiges de $F$ donné selon le cas par la formule correspondante:

- ou bien $\pm d \equiv 2(\bmod 32)$, auquel cas

$$
\left|\widetilde{C l}_{F}^{G}\right|=\left|\widetilde{C l}_{\mathbb{Q}}\right| \frac{\prod_{\mathfrak{p} \mid \infty} d_{\mathfrak{p}}(F / \mathbb{Q}) \cdot \prod_{\mathfrak{p} \nmid \infty} \widetilde{e}_{\mathfrak{p}}(F / \mathbb{Q})}{\left[F: F \cap \mathbb{Q}^{c}\right] \cdot\left(\widetilde{\mathcal{E}}_{\mathbb{Q}}: \widetilde{\mathcal{E}}_{\mathbb{Q}} \cap \mathcal{N}_{F / \mathbb{Q}}\right)}\left|H^{1}\left(G, \widetilde{D l} l_{F}\right)\right| ;
$$

- ou bien $\pm d \equiv 18(\bmod 32)$, auquel cas

$$
\left|\widetilde{C l} l_{F}^{G}\right|=\left|\widetilde{C l}_{\mathbb{Q}}\right| \frac{\prod_{\mathfrak{p} \mid \infty} d_{\mathfrak{p}}(F / \mathbb{Q}) \cdot \prod_{\mathfrak{p} \nmid \infty} \widetilde{e}_{\mathfrak{p}}(F / \mathbb{Q})}{\left[F: F \cap \mathbb{Q}^{c}\right] \cdot\left(\widetilde{\mathcal{E}}_{\mathbb{Q}}: \widetilde{\mathcal{E}}_{\mathbb{Q}} \cap \mathcal{N}_{F / \mathbb{Q}}\right)} .
$$

Preuve. Avec les notations du lemme 4.3.2, nous obtenons

$$
2\left(\mathfrak{B}+\mathfrak{B}^{\sigma}\right)=\widetilde{\operatorname{div}}_{F}(N \beta)-2 \varrho \mathcal{L}=2 \widetilde{\operatorname{div}}_{F}(v)-2 \varrho \mathcal{L},
$$

si bien que

$$
\mathfrak{B}+\mathfrak{B}^{\sigma}=\widetilde{\operatorname{div}}_{F}(v)-\varrho \mathcal{L},
$$

puis

$$
\widetilde{\operatorname{div}}_{F}(\alpha)=\widetilde{\operatorname{div}}_{F}(\beta)-\widetilde{\operatorname{div}}_{F}(v)=2 \mathfrak{B}+\varrho \mathcal{L}-\left(\mathfrak{B}+\mathfrak{B}^{\sigma}\right)-\varrho \mathcal{L}=\mathfrak{B}-\mathfrak{B}^{\sigma} ;
$$

ce qui invite à poser $\mathfrak{A}=\mathfrak{B}^{\sigma}$. Il vient ainsi $\widetilde{\operatorname{div}}_{F}(\alpha)=\mathfrak{A}^{\sigma}-\mathfrak{A} \operatorname{avec} \operatorname{deg}_{F} \mathfrak{A}=$ $-(\varrho / 2) \operatorname{deg}_{F} \mathcal{L}$, soit selon le reste modulo 32 de $\pm d$,

$$
\operatorname{deg}_{F} \mathfrak{A}= \begin{cases}0 & \text { si } \pm d \equiv 2(\bmod 32) \\ -\frac{1}{2} \operatorname{deg}_{F} \mathcal{L} & \text { si } \pm d \equiv 18(\bmod 32) .\end{cases}
$$

Dans le premier cas, le morphisme $\varphi$ est nul, si bien qu'il ne peut être surjectif. Dans le second cas, le degré de la place sauvage $\mathcal{L}$ est égale à 8 si bien que l'entier 2 -adique $-\frac{1}{2} \operatorname{deg}_{F} \mathcal{L}$ est générateur du $\mathbb{Z}_{2}$-module $\operatorname{deg}_{F} D l_{F}$ et que l'image par $\varphi$ de l'unité logarithmique 2 n'est pas nulle. Le morphisme $\varphi$ est donc ici surjectif.

Le paragraphe suivant récapitule enfin ces résultats : 
5. Expression de la formule des classes logarithmiques ambiges sur $\mathbb{Q}$. Les propositions 4.3.3 et 4.4.2 nous suggèrent l'examen préalable du 2 -groupe des classes logarithmiques du corps $\mathbb{Q}$ des nombres rationnels.

5.1. Proposition. Le corps $\mathbb{Q}$ des nombres rationnels est 2-logarithmiquement principal.

Preuve. D'après $\left[J_{3}\right]$, th. 2.3, le 2-groupe $\widetilde{C l}_{\mathbb{Q}}$ des classes logarithmiques de $\mathbb{Q}$ s'identifie au groupe de Galois $\operatorname{Gal}\left(\mathbb{Q}^{\text {lc }} / \mathbb{Q}^{c}\right)$, où $\mathbb{Q}^{c}$ est la $\mathbb{Z}_{2^{-}}$ extension cyclotomique de $\mathbb{Q}$ et $\mathbb{Q}^{\text {lc }}$ la pro-2-extension abélienne maximale de $\mathbb{Q}$ qui est localement cyclotomique (i.e. complètement décomposée sur $\mathbb{Q}^{\text {c }}$ en chacune de ses places). Et comme $\mathbb{Q}^{\text {lc }}$ est contenue dans la pro-2-extension abélienne 2-ramifiée maximale de $\mathbb{Q}$ qui n'est autre ici que $\mathbb{Q}^{c}$ puisque $\mathbb{Q}$ est 2-rationnel au sens de $[\mathrm{GJ}]$ ou de $[\mathrm{MN}]$, il suit que $\mathbb{Q}^{\text {lc }}=\mathbb{Q}^{\mathrm{c}}$, i.e. $\widetilde{C} l_{\mathbb{Q}}=0$.

5.2. ThÉorÈme. Soient F une extension quadratique (CM)-primitivement ramifiée de $\mathbb{Q}$ et d l'entier naturel, supposé sans facteur carré, tel que $F=\mathbb{Q}(\sqrt{ \pm d})$. En désignant par $G$ le groupe de Galois de l'extension $F / \mathbb{Q}$, on trouve le nombre de classes logarithmiques ambiges de $F$ donné selon le cas par la formule suivante:

- $1^{\text {er }}$ cas $:$ pour $\pm d \equiv 1(\bmod 16)$ ou $\pm d \equiv 2(\bmod 32)$, il vient

$$
\left|\widetilde{C l}_{F}^{G}\right|=\frac{\prod_{\mathfrak{p} \mid \infty} d_{\mathfrak{p}}(F / \mathbb{Q}) \cdot \prod_{\mathfrak{p} \nmid \infty} \widetilde{e}_{\mathfrak{p}}(F / \mathbb{Q})}{\left[F: F \cap \mathbb{Q}^{\mathrm{c}}\right] \cdot\left(\widetilde{\mathcal{E}}_{\mathbb{Q}}: \widetilde{\mathcal{E}}_{\mathbb{Q}} \cap \mathcal{N}_{F / \mathbb{Q}}\right)}\left|H^{1}\left(G, \widetilde{D l} l_{F}\right)\right| ;
$$

- $2^{\text {nd }}$ cas $:$ pour $\pm d \not \equiv 1(\bmod 16)$ et $\pm d \not \equiv 2(\bmod 32)$, il vient

$$
\left|\widetilde{C l}_{F}^{G}\right|=\frac{\prod_{\mathfrak{p} \mid \infty} d_{\mathfrak{p}}(F / \mathbb{Q}) \cdot \prod_{\mathfrak{p} \nmid \infty} \widetilde{e}_{\mathfrak{p}}(F / \mathbb{Q})}{\left[F: F \cap \mathbb{Q}^{c}\right] \cdot\left(\widetilde{\mathcal{E}}_{\mathbb{Q}}: \widetilde{\mathcal{E}}_{\mathbb{Q}} \cap \mathcal{N}_{F / \mathbb{Q}}\right)} .
$$

Remarques. 1. Nous rappelons que dans le cas primitif ou imaginaire, l'extension $F / \mathbb{Q}$ est $(C M)$-primitivement ramifiée, si bien que les hypothèses du théorème 5.2 s'avèrent considérablement allégées.

2. Lorsque 2 est norme de l'extension $F / \mathbb{Q}$, où l'entier $d$ satisfait, cette fois-ci, l'une des congruences

$$
\pm d \not \equiv 9(\bmod 16) \quad \text { ou } \quad \pm d \not \equiv 18(\bmod 32)
$$

sa classe engendre le premier groupe de cohomologie $H^{1}\left(G, \widetilde{P l}_{F}\right) \simeq \widetilde{\mathcal{E}}_{\mathbb{Q}} \cap$ $\mathcal{N}_{F / \mathbb{Q}} / N_{F / \mathbb{Q}}\left(\widetilde{\mathcal{E}}_{F}\right)$, puisque son image par $\varphi$ n'est pas nulle. L'extension est donc là aussi $(C M)$-primitivement ramifiée.

3. Malheureusement, en l'absence de l'hypothèse de $(C M)$-primitivité, aucune conclusion ne peut être établie dans le cas général, comme le témoignent ultérieurement certains calculs numériques. 


\section{Application aux extensions quadratiques 2-logarithmique- ment principales de $\mathbb{Q}$}

6.1. Les extensions quadratiques réelles de $\mathbb{Q}$. Les hypothèses sur l'entier $d$ étant conservées, nous considérons dans un premier temps les extensions quadratiques réelles $\mathbb{Q}(\sqrt{d}) / \mathbb{Q}$, et nous donnons une autre formulation de la condition

$$
\prod_{\mathfrak{p} \mid \infty} d_{\mathfrak{p}}(F / \mathbb{Q}) \cdot \prod_{\mathfrak{p} \nmid \infty} \widetilde{e}_{\mathfrak{p}}(F / \mathbb{Q})=\left[F: F \cap \mathbb{Q}^{\mathrm{c}}\right] \cdot\left(\widetilde{\mathcal{E}}_{\mathbb{Q}}: \widetilde{\mathcal{E}}_{\mathbb{Q}} \cap \mathcal{N}_{F / \mathbb{Q}}\right)
$$

précisée en introduction. Cette condition nous conduit à introduire les deux notations suivantes :

- $t$ désigne le nombre de diviseurs premiers impairs de $\mathbb{Q}$, ramifiés dans l'extension $F / \mathbb{Q}$,

- $2^{s}$ est l'indice dans le groupe $\widetilde{\mathcal{E}}_{\mathbb{Q}}$ du sous-groupe $\widetilde{\mathcal{E}}_{\mathbb{Q}} \cap \mathcal{N}_{F / \mathbb{Q}}$ des unités logarithmiques normes dans l'extension $F / \mathbb{Q}$.

Cela posé, la condition (i) devient

$$
\begin{cases}\widetilde{e}_{2}(F / \mathbb{Q})=1 & \text { si } d=2 \\ 2^{t} \times \widetilde{e}_{2}(F / \mathbb{Q})=2 \times 2^{s} & \text { sinon. }\end{cases}
$$

6.1.1. Lemme. La condition (i) est vérifiée lorsque $d=2$ et s'écrit sinon sous la forme

$$
t-s= \begin{cases}1 & \text { sid } d \equiv 1(\bmod 8) \text { ou } d \equiv 2(\bmod 16) \\ 0 & \text { si } d \neq \equiv 1(\bmod 8) \text { et } d \not \equiv 2(\bmod 16)\end{cases}
$$

Preuve. Par définition de l'indice de ramification logarithmique en 2, il vient en effet

$$
\begin{aligned}
\widetilde{e}_{2}(F / \mathbb{Q}) & =\left[\mathbb{Q}_{2}(\sqrt{d}): \mathbb{Q}_{2}(\sqrt{d}) \cap \mathbb{Q}_{2}^{\mathrm{c}}\right] \\
& =\left\{\begin{array}{lll}
1 & \text { si } d \equiv 1 & (\bmod 8) \text { ou } d \equiv 2(\bmod 16), \\
2 & \text { si } d \not \equiv 1 & (\bmod 8) \text { et } d \not \equiv 2(\bmod 16)
\end{array}\right.
\end{aligned}
$$

et le lemme 6.1.1 est ainsi établi.

Ces derniers résultats adjoints au lemme 3.1, nous pouvons à présent dresser la liste des extensions quadratiques réelles 2-logarithmiquement principales de $\mathbb{Q}$. Notant que l'extension $\mathbb{Q}(\sqrt{2})$ est bien 2-logarithmiquement principale; supposons donc désormais $(t \geq 1)$, et distinguons deux cas :

$1^{\mathrm{er}}$ cas $: d \neq 2$ et $(d \equiv 1(\bmod 8)$ ou $d \equiv 2(\bmod 16))$. La condition $t-s=1$ nous conduit à envisager trois possibilités.

- Le cas $t=1$ et $s=0$, auquel cas les extensions obtenues sont

$$
\mathbb{Q}(\sqrt{p}) \text { ou } \mathbb{Q}(\sqrt{2 p}) \quad \text { avec } p \equiv 9(\bmod 16) \quad(\text { cf. } \S 5, \text { rq } 2),
$$


ainsi que certaines des extensions

$$
\mathbb{Q}(\sqrt{p}) \text { ou } \mathbb{Q}(\sqrt{2 p}) \quad \text { avec } p \equiv 1(\bmod 16) .
$$

- Le cas $t=2$ et $s=1$, qui nous donne les extensions

$$
\mathbb{Q}(\sqrt{p q}) \text { ou } \mathbb{Q}(\sqrt{2 p q}) \quad \text { avec } p \equiv \pm 3(\bmod 8) \text { et } q \equiv \pm 3(\bmod 8) ;
$$

$\mathbb{Q}(\sqrt{p q})$ ou $\mathbb{Q}(\sqrt{2 p q}) \quad$ avec $p \equiv-1(\bmod 8)$ et $q \equiv-1(\bmod 8)$.

Dans la dernière situation, en effet, ni -1 , ni -2 ne sont normes, si bien que la condition de $(C M)$-primitivité est vérifiée. Ainsi, placés sous les hypothèses du théorème 5.2 , nous en déduisons les congruences nécessaires : $p \equiv-1(\bmod 16)$ et $q \equiv-9(\bmod 16)$.

- Le cas $t=3$ et $s=2$ enfin, qui nous donne les extensions

$$
\begin{aligned}
\mathbb{Q}(\sqrt{p q r}) \text { ou } \mathbb{Q}(\sqrt{2 p q r}) \quad \text { avec } p & \equiv 3(\bmod 8), q \equiv 5(\bmod 8) \text { et } \\
r & \equiv-1(\bmod 8) .
\end{aligned}
$$

$2^{\text {ième }}$ cas $: d \not \equiv 1(\bmod 8)$ et $d \not \equiv 2(\bmod 16)$. La condition $t-s=0$ ouvre ici deux possibilités seulement :

- ou bien $t=s=1$, auquel cas les extensions obtenues sont $\mathbb{Q}(\sqrt{p})$ ou $\mathbb{Q}(\sqrt{2 p}) \quad$ avec $p \equiv-1(\bmod 8)$ ou $p \equiv \pm 3(\bmod 8)$,

- ou bien $t=s=2$, auquel cas les extensions obtenues sont $\mathbb{Q}(\sqrt{p q})$ ou $\mathbb{Q}(\sqrt{2 p q}) \quad$ avec $\left\{\begin{array}{l}p \equiv 3(\bmod 8) \text { et } q \equiv 5(\bmod 8) \text {, ou } \\ p \equiv \pm 3(\bmod 8) \text { et } q \equiv-1(\bmod 8) .\end{array}\right.$

6.1.2. ThÉORÈme. Les extensions quadratiques réelles 2-logarithmiquement de $\mathbb{Q}$ sont les extensions suivantes (où $p, q, r$ désignent trois premiers impairs distincts arbitraires de $\mathbb{N}$ ):

- $\mathbb{Q}(\sqrt{2})$

- $\mathbb{Q}(\sqrt{p})$ ou $\mathbb{Q}(\sqrt{2 p})$ avec $p \equiv-1(\bmod 8), p \equiv 9(\bmod 16)$ ou $p$ $\equiv \pm 3(\bmod 8)$,

- $\mathbb{Q}(\sqrt{p q})$ ou $\mathbb{Q}(\sqrt{2 p q})$ avec $\left\{\begin{array}{l}p \equiv-1(\bmod 16) \text { et } q \equiv-9(\bmod 16) \text {, ou } \\ p \equiv \pm 3(\bmod 8) \text { et } q \neq 1(\bmod 8),\end{array}\right.$

- $\mathbb{Q}(\sqrt{p q r})$ ou $\mathbb{Q}(\sqrt{2 p q r})$ avec $p \equiv 3(\bmod 8), q \equiv 5(\bmod 8)$ et $r$ $\equiv-1(\bmod 8)$

et les extensions de la forme

- $\mathbb{Q}(\sqrt{d})$ avec $d=p$ ou $d=2 p$ et $p \equiv 1(\bmod 16)$ lorsqu'on peut écrire

$$
-1=N\left(\frac{u^{\prime}+\sqrt{d}}{v^{\prime}}\right) \quad \text { avec }\left\{\begin{array}{l}
u^{\prime} \equiv \pm 7(\bmod 16) \text { pour } d \equiv 1(\bmod 32), \\
u^{\prime} \equiv \pm 1(\bmod 16) \text { pour } d \equiv 17(\bmod 32), \\
u^{\prime} \equiv \pm 3(\bmod 8) \text { pour } d \equiv 2(\bmod 32) .
\end{array}\right.
$$


Preuve. Examinons le cas des extensions $F$ de la forme $\mathbb{Q}(\sqrt{p})$ ou $\mathbb{Q}(\sqrt{2 p})($ avec $p \equiv 1(\bmod 16))$. Conformément à la preuve du lemme 4.3.2, il est possible d'écrire 2 sous la forme $2=N((u+\sqrt{d}) / v)$ avec $\widetilde{v}_{\mathcal{L}}(u+\sqrt{d})$ paire. L'analogue de la preuve du lemme 4.1 montre que -1 est norme dans l'extension $F / \mathbb{Q}$ si et seulement si $(-1 / p)=1$, i.e. $p \equiv 1(\bmod 4)$. Par conséquent, nous pouvons affirmer que dans cette situation $d$ est effectivement norme dans l'extension quadratique $\mathbb{Q}(i) / \mathbb{Q}$, c'est-à-dire puisque l'anneau $\mathbb{Z}[i]$ est principal, qu'il existe deux entiers relatifs $u^{\prime}$ et $v^{\prime}$ tels que l'on ait $\beta^{\prime}=u^{\prime 2}+v^{\prime 2}=d$. L'analogue du lemme 4.2 prouve alors que toutes les $\mathfrak{p}$-valuations logarithmiques de $u^{\prime}+\sqrt{d}$ sont paires en toute place finie modérée $\mathfrak{p}$ de $F$, si bien que le corps quadratique $F=\mathbb{Q}(\sqrt{d})$ est 2logarithmiquement si et seulement si $\widetilde{v}_{\mathcal{L}}\left(u^{\prime}+\sqrt{d}\right)$ est impaire. Bien entendu, les entiers $u^{\prime}$ et $v^{\prime}$ ayant des rôles symétriques, nous pouvons supposer $u^{\prime}$ impair. Trois cas se distinguent alors :

- Lorsque $d=p$ et $p \equiv 1(\bmod 32)$, il est possible de choisir la racine carrée de $p$ congrue à -1 modulo 16 . Notons alors les deux situations suivantes:

- ou bien $u^{\prime}$ peut être choisi congru à -1 modulo 16 , si bien que $\beta^{\prime} \equiv$ $-2(\bmod 16)$ et $F$ n'est pas 2-logarithmiquement principal,

- ou bien $u^{\prime}$ peut être choisi congru à 7 modulo 16 , si bien que $\beta^{\prime} \equiv$ $6(\bmod 16)$ et $F$ est 2-logarithmiquement principal.

- Lorsque $d=p$ et $p \equiv 17(\bmod 32)$, il est possible de choisir la racine carrée de $p$ congrue à 7 modulo 16 . Notons alors les deux situations suivantes :

- ou bien $u^{\prime}$ peut être choisi congru à -1 modulo 16 , si bien que $\beta^{\prime} \equiv$ $6(\bmod 16)$ et $F$ est 2-logarithmiquement principal,

- ou bien $u^{\prime}$ peut être choisi congru à 7 modulo 16 , si bien que $\beta^{\prime} \equiv$ $-2(\bmod 16)$ et $F$ n'est pas 2-logarithmiquement principal.

- Lorsque $d=2 p$ et $p \equiv 1(\bmod 16), u^{\prime}$ et $v^{\prime}$ ayant des rôles symétriques, les $\mathcal{L}$-valuations logarithmiques attachées à la place sauvage $\mathcal{L}$ de $F$ des nombres $\beta=u^{\prime}+\sqrt{d}$ et $v^{\prime}+\sqrt{d}$ sont de même parité. En particulier, $\widetilde{v}_{\mathcal{L}}(\beta)$ est impaire si et seulement si $u^{\prime} \equiv \pm 3(\bmod 8)$.

Le reste est alors immédiat.

Remarques. 1. Dans la dernière situation, la condition nécessaire et suffisante obtenue porte uniquement sur les congruences puisque nous sommes assurés de l'existence de $u+\sqrt{d}$ et $u^{\prime}+\sqrt{d}$. Elle est donc tout à fait explicite et caractérise parfaitement les corps de nombres 2-logarithmiquement principaux de la forme $\mathbb{Q}(\sqrt{p})$ ou $\mathbb{Q}(\sqrt{2 p})$ avec $p \equiv 1(\bmod 16)$.

2 . Une sous-extension d'un corps de nombres 2-logarithmiquement principal n'est pas forcément 2-logarithmiquement principale. En effet, posons 
$K=\mathbb{Q}(\sqrt{p})$ et $L=\mathbb{Q}(i, \sqrt{p})$, où $p$ est un nombre premier congru à 3 modulo 8. Alors l'extension $L$ est 2-logarithmiquement principale (cf. [Th]), bien que le corps de nombres $K$ ne le soit pas.

3. Selon Browkin et Schinzel (cf. [BS], th. 2), les extensions quadratiques dont le 2-Sylow du noyau hilbertien est trivial sont celles de la forme :

- $\mathbb{Q}(\sqrt{2})$,

- $\mathbb{Q}(\sqrt{p})$ ou $\mathbb{Q}(\sqrt{2 p})$ avec $p \equiv 3(\bmod 8)$ ou $p \equiv 5(\bmod 8)$,

- $\mathbb{Q}(\sqrt{p})$ avec $p \equiv 1(\bmod 8), p$ ne pouvant pas s'écrire sous la forme $u^{2}-2 v^{2}$ avec $u>0, u \equiv 1(\bmod 4)$ et $v \equiv 0(\bmod 4)$,

- $\mathbb{Q}(\sqrt{p q})$ avec $p \equiv 3(\bmod 8)$ et $q \equiv 3(\bmod 8)$.

Cela nous conduit à la proposition suivante :

6.1.3. SCOLIE. Les extensions quadratiques réelles dont le 2-Sylow du noyau hilbertien est trivial sont 2-logarithmiquement principales.

Preuve. Examinons le seul cas douteux des extensions quadratiques réelles de la forme $F=\mathbb{Q}(\sqrt{p})$ où $p \equiv 1(\bmod 16)$ et dont le 2-Sylow du noyau hilbertien est trivial. D'après les résultats de $\mathrm{H}$. Thomas (cf. [Th], p. 472, Prop. 1, cas 1), le 2-groupe des classes logarithmiques de l'extension biquadratique $K=\mathbb{Q}(i, \sqrt{p})$ est alors cyclique d'ordre 2. En particulier, si $\Delta$ désigne le groupe de Galois de l'extension $K / F$, il coïncide avec son 2-groupe ambige $\widetilde{C l}{ }_{K}^{\Delta}$. La formule des classes logarithmiques ambiges nous donne donc la minoration suivante :

$$
\left|\widetilde{C l}_{F}\right| \geq \frac{\left(\widetilde{\mathcal{E}}_{F}: \widetilde{\mathcal{E}}_{F} \cap \mathcal{N}_{K / F}\right)}{2},
$$

puisque le conoyau du morphisme $\left[H^{1}\left(\Delta, \widetilde{P l}_{K}\right) \rightarrow H^{1}\left(\Delta, \widetilde{D l}_{K}\right)\right]$ déduit de la suite exacte

$$
1 \rightarrow \widetilde{P l}_{K} \rightarrow \widetilde{D l}_{K} \rightarrow \widetilde{C l}_{K} \rightarrow 1
$$

est au plus d'ordre 2. Si $\varepsilon$ désigne une unité fondamentale de $F,-1$ et $\varepsilon$ sont deux générateurs du 2-groupe des unités logarithmiques de $F$. Comme $K$ est totalement imaginaire, -1 ne peut être norme dans l'extension $K / F$. Supposons en revanche que $\varepsilon$ le soit, auquel cas son conjugué $\bar{\varepsilon}$ et leur produit $\varepsilon \bar{\varepsilon}=-1$ sont tous deux normes dans l'extension $K / F$; ce qui ne peut être. Une vérification analogue montre plus précisément que les classes de -1 et $\varepsilon$ sont distinctes modulo $\widetilde{\mathcal{E}}_{F} \cap \mathcal{N}_{K / F}$. L'indice $\left(\widetilde{\mathcal{E}}_{F}: \widetilde{\mathcal{E}}_{F} \cap \mathcal{N}_{K / F}\right)$ est ainsi égal à 4 et le corps quadratique $F$ n'est pas 2-logarithmiquement principal.

Remarque. En revanche, aucune réciproque n'est envisageable. En effet, l'extension quadratique $\mathbb{Q}(\sqrt{p q})$ où $p$ et $q$ désignent deux nombres premiers distincts congrus à 5 modulo 8 est primitivement ramifiée et 2logarithmiquement principale, bien que la 2-partie de son noyau hilbertien ne soit pas triviale. 
6.1.4. Exemples numériques. Dans ces exemples, nous illustrons la situation des corps de nombres

$$
\mathbb{Q}(\sqrt{p}) \text { et } \mathbb{Q}(\sqrt{2 p}) \quad \text { avec } p \equiv 1(\bmod 16) .
$$

EXemple 1. Lorsque $F=\mathbb{Q}(\sqrt{17})$, nous considérons $\beta^{\prime}=-1+\sqrt{17}$ un élément de norme $-4^{2}$. Comme $u^{\prime}=-1, F$ est 2-logarithmiquement principal.

EXEMPLE 2. Lorsque $F=\mathbb{Q}(\sqrt{113})$, nous considérons le nombre $\beta^{\prime}=7+$ $\sqrt{113}$ de norme $-8^{2}$. Comme $u^{\prime} \equiv \pm 7(\bmod 16), F$ n'est pas 2-logarithmique-

ment principal.

EXEMPle 3. Lorsque $F=\mathbb{Q}(\sqrt{34})$, l'unité logarithmique -1 est norme de l'élément $\alpha^{\prime}=(5+\sqrt{34}) / 3$. Comme $u^{\prime} \equiv \pm 3(\bmod 8)$, l'extension $F$ est ici 2-logarithmiquement principale.

EXEMPLE 4. Lorsque $F=\mathbb{Q}(\sqrt{226})$, l'unité logarithmique -1 est norme de l'élément $\beta^{\prime}=15+\sqrt{226}$. Comme $u^{\prime} \equiv \pm 1(\bmod 8), F=\mathbb{Q}(\sqrt{226})$ n'est pas 2-logarithmiquement principal.

6.2. Les extensions quadratiques imaginaires. Les hypothèses sur l'entier $d$ étant conservées, nous considérons à présent les extensions quadratiques imaginaires $\mathbb{Q}(\sqrt{-d}) / \mathbb{Q}$. De façon analogue, nous démontrons successivement les résultats suivants :

6.2.1. Lemme. La condition (i) est vérifiée lorsque $d=2$ et s'écrit sinon sous la forme

(i) $)^{\prime \prime} \quad t-s=\left\{\begin{array}{lll}0 & \text { si } d \equiv-1(\bmod 8) \text { ou } d \equiv-2(\bmod 16), \\ 1 & \text { si } d \neq 1(\bmod 8) \text { et } d \not \equiv-2(\bmod 16),\end{array}\right.$

où s peut être l'entier 1 ou 2 .

6.2.2. ThÉORÈme. Les extensions quadratiques imaginaires 2-logarithmiquement de $\mathbb{Q}$ sont les extensions suivantes (où $p$ et $q$ désignent deux nombres premiers impairs distincts arbitraires de $\mathbb{N})$ :

- $\mathbb{Q}(\sqrt{-2})$,

- $\mathbb{Q}(i)$

- $\mathbb{Q}(\sqrt{-p})$ ou $\mathbb{Q}(\sqrt{-2 p})$ avec $p \equiv \pm 3(\bmod 8)$ ou $p \equiv-9(\bmod 16)$,

- $\mathbb{Q}(\sqrt{-p q})$ ou $\mathbb{Q}(\sqrt{-2 p q})$ avec $p \equiv 3(\bmod 8)$ et $q \equiv 5(\bmod 8)$.

R e marque. Selon Browkin et Schinzel (cf. [BS], th. 4), les extensions quadratiques imaginaires dont le 2-Sylow du noyau hilbertien est trivial sont celles de la forme :

- $\mathbb{Q}(\sqrt{-2})$ ou $\mathbb{Q}(i)$

- $\mathbb{Q}(\sqrt{-p})$ ou $\mathbb{Q}(\sqrt{-2 p})$ avec $p \equiv \pm 3(\bmod 8)$, 
- $\mathbb{Q}(\sqrt{-p})$ avec $p \equiv-1(\bmod 8)$,

- $\mathbb{Q}(\sqrt{-p q})$ avec $p \equiv 3(\bmod 8)$ et $q \equiv 5(\bmod 8)$.

Nous constatons alors :

- que toute extension quadratique imaginaire dont le 2-Sylow du noyau hilbertien est trivial, est 2-logaritmiquement principale, dès qu'elle est 2primitivement ramifiée sur $\mathbb{Q}$,

- qu'un corps de nombres imaginaire dont la 2-partie du noyau hilbertien est trivial, n'est pas nécessairement 2-logarithmiquement principal (comme l'illustre l'exemple $F=\mathbb{Q}(\sqrt{-31})$ ).

\section{Références}

[BT] H. Bass and J. Tate, The Milnor ring of a global field, dans: Algebraic K-Theory II, Lecture Notes in Math. 342, Springer, 1973, 349-428.

[BS] J. Browkin and A. Schinzel, On Sylow 2-subgroups of $K_{2}\left(\mathcal{O}_{F}\right)$ for quadratic number fields F, J. Reine Angew. Math. 331 (1982), 104-113.

[GJ] G. Gras et J.-F. Jaulent, Sur les corps de nombres réguliers, Math. Z. 202 (1989), 343-365.

$\left[\mathrm{J}_{1}\right] \quad$ J.-F. Jaulent, La théorie de Kummer et le $K_{2}$ des corps de nombres, Sém. Théor. Nombres Bordeaux 2 (1990), 377-411.

$\left[\mathrm{J}_{2}\right] \quad$ - Sur le noyau sauvage des corps de nombres, Acta Arith. 67 (1994), 335-348.

$\left[\mathrm{J}_{3}\right] \quad$ - Classes logarithmiques des corps de nombres, J. Théor. Nombres Bordeaux 6 (1994), 301-325.

[JN] J.-F. Jaulent and T. Nguyen Quang Do, Corps p-rationnels, corps p-réguliers, et ramification restreinte, ibid. 5 (1994), 343-363.

[MN] A. Movahhedi and T. Nguyen Quang Do, Sur l'arithmétique des corps de nombres p-rationnels, dans: Séminaire de Théorie des Nombres, Paris 1987/1988, Progr. in Math. 81, Birkhäuser, 1990, 155-200.

[Qi] H. Qin, Computation of $K_{2} \mathbb{Z}[\sqrt{-6}]$, J. Pure Appl. Algebra 96 (1994), 133-146.

[Sc] P. Schneider, Über gewisse Galoiscohomologiegruppen, Math. Z. 168 (1979), 181-205.

[Sk] M. Skałba, Generalization of Thue's theorem and computation of the group $K_{2} \mathcal{O}_{F}$, J. Number Theory 46 (1994), 303-322.

[Th] H. Thomas, Trivialité du 2-rang du noyau hilbertien, J. Théor. Nombres Bordeaux 6 (1994), 459-483.

Laboratoire de Mathématiques Pures

Université de Bordeaux I

351, cours de la Libération

33405 Talence Cedex, France

E-mail: soriano@math.u-bordeaux.fr 